\title{
QUADRUPLE INTEGRAL EQUATIONS AND OPERATORS OF FRACTIONAL INTEGRATION
}

\author{
by M. IFTIKHAR AHMAD
}

(Received 2 December, 1969)

Cooke [1] modified a technique used by Erdélyi and Sneddon [2] to solve triple integral equations of a certain type. In this paper, we extend this method to solve the quadruple integral equations

$$
\begin{array}{ll}
L_{1}(\alpha, \rho) \equiv \int_{0}^{\infty} \xi^{-2 \alpha} \psi(\xi) J_{v}(\rho \xi) d \xi=F_{1}(\rho) & (0<\rho<a), \\
L_{2}(\beta, \rho) \equiv \int_{0}^{\infty} \xi^{-2 \beta} \psi(\xi) J_{v}(\rho \xi) d \xi=G_{2}(\rho) & (a<\rho<b), \\
L_{3}(\alpha, \rho) \equiv \int_{0}^{\infty} \xi^{-2 \alpha} \psi(\xi) J_{v}(\rho \xi) d \xi=F_{3}(\rho) & (b<\rho<c), \\
L_{4}(\beta, \rho) \equiv \int_{0}^{\infty} \xi^{-2 \beta} \psi(\xi) J_{v}(\rho \xi) d \xi=G_{4}(\rho) & (\rho>c),
\end{array}
$$

where $F_{1}, G_{2}, F_{3}$ and $G_{4}$ are prescribed functions of $\rho$ and $\psi(\zeta)$ is to be determined. With no loss of generality we shall assume that $G_{2}(\rho) \equiv 0, G_{4}(\rho) \equiv 0$.

1. Operators. We recall here a few definitions and properties of the operators used in solving the integral equations (1). Cooke [1] has defined $\dagger$ the operators ${ }_{a}^{b} I_{n, \alpha}$ and ${ }_{c}^{d} K_{n, \alpha}$ by the formulae

$$
\begin{array}{ll}
{ }_{a}^{b} I_{\eta, \alpha} f(x)=\frac{2 x^{-2 \alpha-2 \eta}}{\Gamma(\alpha)} \int_{a}^{b}\left(x^{2}-u^{2}\right)^{\alpha-1} u^{2 \eta+1} f(u) d u & (\alpha>0), \\
{ }_{a}^{b} I_{\eta, \alpha} f(x)=\frac{x^{-2 \eta-2 \alpha-1}}{\Gamma(1+\alpha)} \frac{d}{d x} \int_{a}^{b}\left(x^{2}-u^{2}\right)^{\alpha} u^{2 \eta+1} f(u) d u & (-1<\alpha<0), \\
{ }_{c}^{d} K_{\eta, \alpha} f(x)=\frac{2 x^{2 \eta}}{\Gamma(\alpha)} \int_{c}^{d}\left(u^{2}-x^{2}\right)^{\alpha-1} u^{-2 \alpha-2 \eta+1} f(u) d u & (\alpha>0), \\
{ }_{c}^{d} K_{\eta, \alpha} f(x)=-\frac{x^{2 \eta-1}}{\Gamma(1+\alpha)} \frac{d}{d x} \int_{c}^{d}\left(u^{2}-x^{2}\right)^{\alpha} u^{-2 x-2 \eta+1} f(u) d u & (-1<\alpha<0) .
\end{array}
$$

For $\alpha=0$, these are just the identity operators. Note that with these definitions ${ }_{0}^{x} I_{\eta, \alpha}$ and ${ }_{x}^{\infty} K_{\eta, \alpha}$ are simply the Erdélyi-Kober operators [5]. In these cases we will drop the indices on the left and write them as $I_{\eta, \alpha}$ and $K_{\eta, a}$. We also observe that (2), (3) make sense if $b<x$ and similarly (4), (5) are defined only if $c>x$.

$\dagger$ Cooke uses $\left(\begin{array}{c}b \\ a\end{array}\right) I_{\eta, \alpha},\left(\begin{array}{c}d \\ c\end{array}\right) K_{\eta, \alpha}$, but our notation seems convenient. 
The modified operator $S_{\eta, \alpha}$ of the Hankel transforms is defined by

$$
S_{\eta, \alpha} f(x)=2^{\alpha} x^{-\alpha} \int_{0}^{\infty} \xi^{1-\alpha} J_{2 \eta+\alpha}(x \xi) f(\tilde{\zeta}) d \xi
$$

Sneddon [4] has shown the following relations between the Erdélyi-Kober and Hankel operators.

$$
\begin{aligned}
I_{\eta+\alpha, \beta} S_{\eta, \alpha} & =S_{\eta, \alpha+\beta}, \\
K_{\eta, \alpha} S_{\eta+\alpha, \beta} & =S_{\eta, \alpha+\beta}, \\
S_{\eta+\alpha, \beta} S_{\eta, \alpha} & =I_{\eta, \alpha+\beta}, \\
S_{\eta, \alpha} S_{\eta+\alpha, \beta} & =K_{\eta, \alpha+\beta},
\end{aligned}
$$

provided that the conditions for the existence of the various operations are satisfied. The inverse operators are

$$
\begin{aligned}
{ }_{a}^{b} I_{\eta, \alpha}^{-1} & ={ }_{a}^{b} I_{\eta+\alpha,-\alpha}, \\
{ }_{c}^{d} K_{\eta, \alpha}^{-1} & ={ }_{c}^{d} K_{\eta+\alpha,-\alpha}, \\
S_{\eta, \alpha}^{-1} & =S_{\eta+\alpha,-\alpha} .
\end{aligned}
$$
operators.

We require two lemmas also given by Cooke [1], which define the product of pairs of

LemmA A. Let ${ }_{a}^{b} I_{\eta, \alpha},{ }_{d}^{x} I_{\eta, \alpha}^{-1}$ be operators as defined in (2), (3) and (11). Then

$$
{ }_{d}^{x} I_{\eta, \alpha a}^{-1 b} I_{\eta, \alpha} f(x)=\frac{2 \sin \pi \alpha}{\pi} x^{-2 \eta}\left(x^{2}-d^{2}\right)^{-\alpha} \int_{a}^{b} \frac{\left(d^{2}-t^{2}\right)^{\alpha} t^{2 n+1} f(t)}{x^{2}-t^{2}} d t
$$

provided that $x>d \geqq b>a$.

Lemma B. Let ${ }_{a}^{b} K_{\eta, a}{ }_{x}^{d} K_{\eta, a}^{-1}$ be operators as defined in (4), (5), and (12). Then

$$
{ }_{x}^{d} K_{\eta, \alpha \alpha}^{-1 b} K_{\eta, \alpha} f(x)=\frac{2 \sin \pi \alpha}{\pi} x^{2 \eta+2 \alpha}\left(d^{2}-x^{2}\right)^{-\alpha} \int_{a}^{b} \frac{\left(t^{2}-d^{2}\right)^{\alpha} t^{-2 \alpha-2 \eta+1} f(t)}{t^{2}-x^{2}} d t,
$$

provided that $x<d \leqq a<b$.

2. Solution of the equations (1). We transform the equations (1) into a form to which the operational theory is applicable by substituting

$$
\psi(\xi)=\xi A(\xi), \quad f_{l}(\rho)=(2 / \rho)^{2} F_{i}(\rho)
$$


by means of this we get

$$
\begin{array}{ll}
L_{1}(\alpha, \rho) \equiv 2^{2 \alpha} \rho^{-2 \alpha} \int_{0}^{\infty} \xi^{1-2 \alpha} A(\xi) J_{v}(\rho \xi) d \xi=f_{1}(\rho) & (0<\rho<a), \\
L_{2}(\beta, \rho) \equiv 2^{2 \beta} \rho^{-2 \beta} \int_{0}^{\infty} \xi^{1-2 \beta} A(\xi) J_{v}(\rho \xi) d \xi=0 & (a<\rho<b), \\
L_{3}(\alpha, \rho) \equiv 2^{2 \alpha} \rho^{-2 \alpha} \int_{0}^{\infty} \xi^{1-2 \alpha} A(\xi) J_{v}(\rho \xi) d \xi=f_{3}(\rho) & (b<\rho<c), \\
L_{4}(\beta, \rho) \equiv 2^{2 \beta} \rho^{-2 \beta} \int_{0}^{\infty} \xi^{1-2 \beta} A(\xi) J_{v}(\rho \xi) d \xi=0 & (\rho>c) .
\end{array}
$$

Let $I_{1}$ denote the interval $(0, a), I_{2}$ the interval $(a, b), I_{3}$ the interval $(b, c)$ and $I_{4}$ the interval $(c, \infty)$. For a function $f$ in $L_{2}(0, \infty)$ we shall write $f_{1}+f_{2}+f_{3}+f_{4}$, where

$$
f_{i}=f \quad \text { on } I_{i} \text { and }=0 \quad \text { on } I_{j} \quad(i, j=1,2,3,4 ; i \neq j)
$$

and similarly for $g$. Using the $S$-operator defined in (6), we see that the integral equations (17) reduce to the form

$$
\begin{aligned}
& S_{\frac{1}{2} \nu-\alpha, 2 \alpha} A(\rho)=f(\rho), \\
& S_{\frac{1}{2}-\beta, 2 \beta} A(\rho)=g(\rho) .
\end{aligned}
$$

Here $f_{1}$ and $f_{3}$ are prescribed, $g_{2}=0=g_{4}$ but $g_{1}, f_{2}, g_{3}$ and $f_{4}$ are to be determined. Let us take as trial solution

$$
A(\rho)=S_{\frac{1}{2} \nu+\beta,-\alpha-\beta} l(\rho) .
$$

Substituting this value of $A(\rho)$ in (18), (19) and using formulas (9), (10) we have

$$
\begin{aligned}
& f=I_{\frac{1}{2} v+\beta, \alpha-\beta} l, \\
& g=K_{\frac{1}{2} \nu-\beta, \beta-\alpha} l .
\end{aligned}
$$

Also, we have

$$
\begin{aligned}
l & =I_{\frac{1}{2} v+\beta, \alpha-\beta}^{-1} f \\
& =K_{\frac{1}{2} \nu-\beta, \beta-\alpha}^{-1} g .
\end{aligned}
$$

We proceed to determine $l$. The subscripts on all the operators will be dropped for brevity sake. All $I$ 's will be supposed to have subscripts $\frac{1}{2} v+\beta, \alpha-\beta$ understood and all $K$ 's to have $\frac{1}{2} v-\beta, \beta-\alpha$.

Evaluating (23) on $I_{1}$, we get

$$
l_{1}={ }_{0}^{\rho} I^{-1} f_{1}
$$

Taking (24) on $I_{4}$, we have

$$
l_{4}={ }_{\rho}^{\infty} K^{-1} g_{4}=0
$$


Evaluate (22) on $I_{2}$; then

$$
{ }_{\rho}^{b} K l_{2}+{ }_{b}^{c} K l_{3}+{ }_{c}^{\infty} K l_{4}=0,
$$

which gives

$$
l_{2}=-{ }_{\rho}^{b} K^{-1}{ }_{b}^{c} K l_{3}
$$

Applying Lemma B, we have

$$
l_{2}(\rho)=-\frac{2 \sin \pi(\beta-\alpha)}{\pi} \rho^{v-2 \alpha}\left(b^{2}-\rho^{2}\right)^{\alpha-\beta} \int_{b}^{c} \frac{\left(t^{2}-b^{2}\right)^{\beta-\alpha} t^{-v+2 \alpha+1 / l_{3}(t)}}{t^{2}-\rho^{2}} d t .
$$

Finally, evaluating (21) on $I_{3}$, we have

$$
l_{3}={ }_{b}^{\rho} I^{-1} f_{3}-{ }_{b}^{\rho} I^{-1}{ }_{0}^{a} I l_{1}-{ }_{b}^{\rho} I^{-1}{ }_{a}^{b} I l_{2} .
$$

Since $f_{3}$ and $l_{1}$ are known functions, the function

$$
d(\rho)={ }_{b}^{\rho} I^{-1} f_{3}(\rho)-{ }_{b}^{\rho} I^{-1}{ }_{0}^{a} I_{1}
$$

is known. Applying Lemma A to the last term on the right-hand side of (29) and substituting (28), (30) in that equation, we obtain

$$
\begin{aligned}
l_{3}(\rho)= & d(\rho)+\frac{2 \sin \pi(\alpha-\beta)}{\pi} \rho^{-\nu-2 \beta}\left(\rho^{2}-b^{2}\right)^{\beta-\alpha} \\
& \times \int_{a}^{b}\left(b^{2}-y^{2}\right)^{\alpha-\beta} y^{\nu+2 \beta+1}\left\{\frac{2 \sin \pi(\beta-\alpha)}{\pi} y^{\nu-2 \alpha}\left(b^{2}-y^{2}\right)^{\alpha-\beta}\right. \\
& \left.\times \int_{b}^{c} \frac{\left(t^{2}-b^{2}\right)^{\beta-\alpha} t^{-v+2 \alpha+1} l_{3}(t)}{t^{2}-y^{2}} d t\right\} \frac{1}{\rho^{2}-y^{2}} d y .
\end{aligned}
$$

Inverting the order of integration, we get

$$
\begin{aligned}
l_{3}(\rho)=d(\rho) & -\frac{4 \sin ^{2} \pi(\alpha-\beta)}{\pi^{2}} \int_{b}^{c}\left\{\rho^{-v-2 \beta}\left(\rho^{2}-b^{2}\right)^{\beta-\alpha}\right. \\
& \times\left(t^{2}-b^{2}\right)^{\beta-\alpha} t^{-v+2 \alpha+1} \int_{a}^{b}\left(b^{2}-y^{2}\right)^{2(\alpha-\beta)} y^{2 v-2 \alpha+2 \beta+1} \\
& \left.\times \frac{1}{\left(t^{2}-y^{2}\right)\left(\rho^{2}-y^{2}\right)} d y\right\} l_{3}(t) d t .
\end{aligned}
$$


Putting $4 \pi^{-2} \sin ^{2} \pi(\alpha-\beta)=-\lambda$, and the expression within the curly brackets equal to $K(\rho, t)$, we obtain

$$
l_{3}(\rho)=d(\rho)+\lambda \int_{b}^{c} K(\rho, t) l_{3}(t) d t
$$

which is a Fredholm's integral equation of the second kind and can be solved by known methods. The equations (25), (26), (28) and (33) completely determine $l$ and our problem is formally solved.

Acknowledgement. I am indebted to Professor W. A. Al-Salam for his encouragement and help during the preparation of this paper.

\section{REFERENCES}

1. J. C. Cooke, The solution of triple integral equations in operational form, Quart. J. Mech. Appl. Math. 18 (1965), 57-72.

2. A. Erdélyi and I. N. Sneddon, Fractional integration and dual integral equations, Canad. J. Math. 14 (1962), 685-693.

3. H. Kober, On fractional integrals and derivatives, Quart. J. Math. (1), 11 (1940), 193-211.

4. I. N. Sneddon, Mixed boundary value problems in potential theory (North-Holland Publishing Company, Amsterdam, 1966).

Department of Mathematics

University of the Punjab (New Campus)

Lahore, Punjab

PAKISTAN 